\title{
Solution de branche pour les cabinets médicaux
}

\section{Sonia Barbosa ${ }^{a}$, Michel Matter}

a MHS, experte, département Prestations et développement professionnel de la FMH

b Dr méd., vice-président de la FMH et responsable du département Prestations et développement professionnel

Les entreprises assurées selon la LAA doivent appliquer les prescriptions de la directive de la Commission fédérale de coordination pour la sécurité au travail (CFST) [1]. Pour répondre à cette obligation légale, la FMH a élaboré une solution de branche pour le secteur de la médecine ambulatoire, qui permettra aux médecins-employeurs de répondre à leurs obligations légales de manière accessible, interactive et adaptée à la réalité du terrain.

\section{Introduction}

Une solution de branche est un système de sécurité qui permet à l'employeur de couvrir tous les éléments présents dans son entreprise qui peuvent potentiellement poser un risque de sécurité tant pour le personnel que pour la structure. La solution de branche est une réponse à la méthode MSST qui a pour but de rassembler les exigences essentielles en matière de sécurité au travail et de protection de la santé [2]. Tel que son nom l'indique, c'est une solution qui

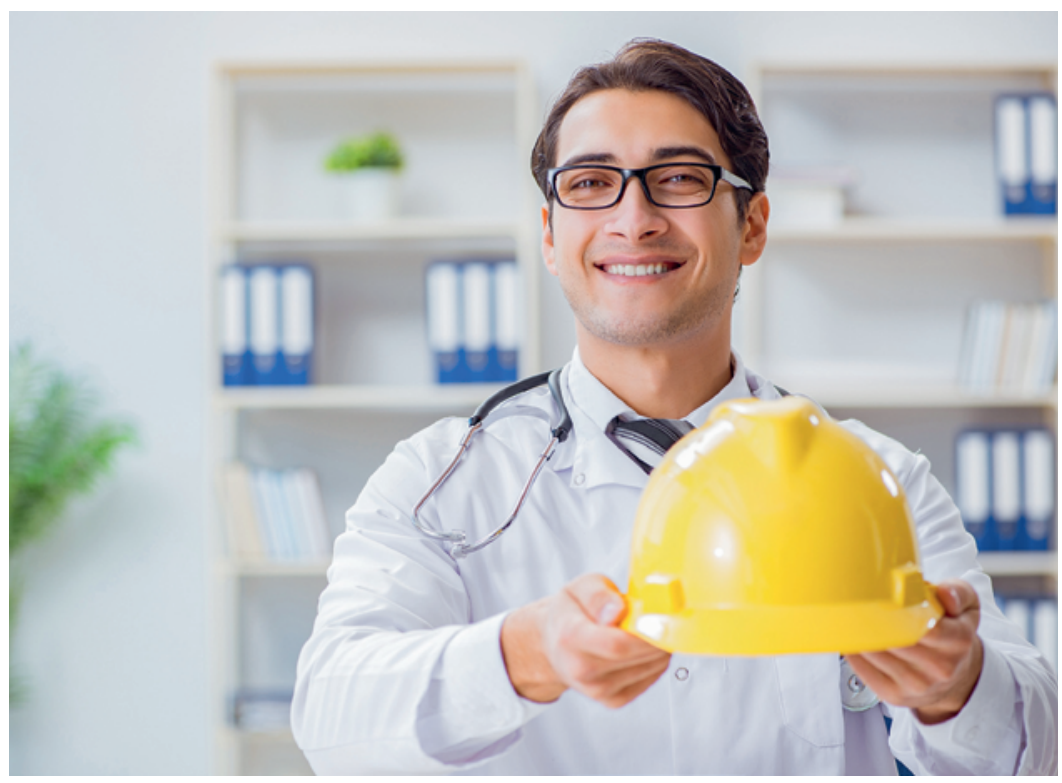

Même si le casque de protection n'est généralement pas nécessaire au cabinet, les médecins doivent aussi assurer la sécurité de leurs collaboratrices et collaborateurs. doit être applicable à l'ensemble de la branche (économique) concernée, et qui est généralement initiée par la faîtière ou l'association de la branche en question. Pour la médecine humaine, il y a une distinction à faire entre le domaine hospitalier et le domaine ambulatoire. Pour le domaine hospitalier, c'est la solution de

Concrètement, pour le cabinet médical, il s'agira principalement de définir un-e "préposé-e à la sécurité».

branche $\mathrm{H}+$ qui fait foi, pour le domaine ambulatoire, ce sera la solution de branche élaborée par l'Association suisse pour la sécurité au travail et la protection de la santé et la FMH qui fera foi, dès l'été 2021. Dans un tel projet d'envergure nationale, les spécificités et les besoins en matière de sécurité des différentes spécialités médicales ont dû être pris en compte afin d'avoir une solution proche du terrain, élaborée par des médecins, pour les médecins, tout en évitant les contraintes et les frais superflus.

\section{La sécurité au travail: une obligation légale}

Toutes les branches économiques sont concernées par l'obligation légale d'avoir une solution de branche depuis son entrée en vigueur en l'an 2000. Les cabinets médicaux ont longtemps été épargnés, le focus étant sur les plus grandes institutions médico-sociales comme les hôpitaux et les homes qui eux devaient être conformes à la loi. Une vingtaine d'années plus tard, la réalité est tout autre; d'une part les cabinets se com- 
plexifient, mais ils sont également de plus en plus grands, ce qui les place dans le viseur des inspectorats cantonaux du travail. Bien que la base légale fédérale soit appliquée différemment dans les cantons, les obligations sont les mêmes et s'appliquent à tous.

\section{Quelles seront mes obligations en tant que médecin-employeur?}

"Quelle que soit leur branche, les employeurs sont tenus de garantir la sécurité au travail et la protection de la santé au sein de leur entreprise [...] C'est sur cette base que la directive MSST concrétise l'obligation incombant à l'employeur de faire appel à des spécialistes de la sécurité au travail au sens de l'ordonnance sur la prévention des accidents (OPA) ainsi que les mesures destinées à promouvoir la prévention systématisée des

\section{La solution de branche a été élaborée dans le but de rester proche du terrain, d'être abordable.}

accidents et maladies professionnelles et la protection de la santé.» [3] Concrètement, pour le cabinet médical, il s'agira principalement de définir un-e «préposé-e à la sécurité» (PàS). Cette personne se familiarisera avec les bases de la sécurité au travail et de la protection de la santé et sera encouragée à accomplir ses tâches de PàS à l'aide de la solution de branche, ce qui lui permettra de planifier les mesures et actions nécessaires à mettre en œuvre dans le cabinet médical [4]. En souscrivant à la solution de branche, le cabinet médical respecte ses responsabilités légales avec une solution de branche certifiée par la CFST.

\section{Est-ce que la solution de branche est adaptée à mon cabinet?}

Une solution de branche se doit d'avoir un spectre aussi large que possible, mais aussi applicable que nécessaire. La solution de branche est structurée sous forme modulaire. Chaque module comporte des sous-chapitres qui couvrent les dangers retrouvés dans les cabinets médicaux. Lors de son élaboration, il a été important d'intégrer les contributions d'un groupe de travail interdisciplinaire composé de méde- cins, en tant qu'organe consultatif et participatif. Ce sont les médecins eux-mêmes qui ont apporté leurs contributions aux modules sécuritaires, mais qui ont également mis leurs différents cabinets à disposition pour pouvoir tester le transfert de la théorie à la pratique lorsque les modules étaient en phase préfinale d'élaboration. Ce modus operandi a pour but de garantir des modules sécuritaires clairs et simples à utiliser. Bien évidemment, pour couvrir tous les domaines de la médecine ambulatoire, il faut couvrir un spectre très large, tout en s'assurant que les informations nécessaires pour chacun restent facilement accessibles. Grâce au logiciel informatique PREVITAR, chaque cabinet médical pourra choisir les modules et chapitres applicables à lui-même, et définir son propre «livre de sécurité» de manière interactive grâce à une interface informatique facile à utiliser. Cette mise en œuvre personnalisée permettra d'accompagner les préposés à la sécurité au travail du cabinet dans l'identification des dangers existants et dans la planification des mesures nécessaires dans leurs domaines de compétence [5].

\section{Conclusion}

La solution de branche pour les cabinets médicaux est le fruit d'un travail de deux ans et demi, initié par les sociétés cantonales de médecine. C'est un aboutissement qui fait suite à une vaste étude de la situation au niveau de la médecine ambulatoire, qui a cherché à trouver des directives existantes sur lesquelles se baser, et qui a cherché des solutions existantes auprès des partenaires afin d'éviter les doublons administratifs. Elle a été élaborée dans le but de rester proche du terrain, d'être abordable, d'être accessible pour le personnel du cabinet, et de bénéficier tout particulièrement aux membres de la FMH.

\footnotetext{
Références

1 https://ekas.ch/index-fr.php?frameset=20: directive MSST

2 https://ekas.ch/index-fr.php?frameset=20: la méthode MSST

3 www.suva.ch/fr-ch/prevention/themes-selon-les-branches/ autres-branches

4 www.arbeitssicherheitschweiz.ch/fr/offres/cours/pas-membres

5 www.arbeitssicherheitschweiz.ch/fr/offres/solution-branche: solution de branche de l'Association suisse pour la sécurité au travail et la protection de la santé
}

Crédits photo

(c) Elnur | Dreamstime.com (image symbolique) 